\title{
The electron energy distribution during HF pumping, a picture painted with all colors
}

\author{
B. Gustavsson ${ }^{1}$, T. Sergienko ${ }^{2}$, M. .J Kosch ${ }^{3}$, M. T. Rietveld ${ }^{4}$, B. U. E. Brändström ${ }^{2}$, T. B. Leyser ${ }^{5}$, B. Isham ${ }^{6}$, \\ P. Gallop ${ }^{7}$, T. Aso ${ }^{1}$, M. Ejiri ${ }^{1}$, T. Grydeland ${ }^{8},{ }^{\circ}$ A. Steen ${ }^{9}$, C. LaHoz ${ }^{8}$, K. Kaila ${ }^{10}$, J. Jussila ${ }^{10}$, and H. Holma ${ }^{10}$ \\ ${ }^{1}$ National Institute of Polar Research, Tokyo, Japan \\ ${ }^{2}$ Swedish Institute of Space Physics, Kiruna, Sweden \\ ${ }^{3}$ Department of Communication Systems, Lancaster University, Lancaster, England, UK \\ ${ }^{4}$ EISCAT, Ramfjordmoen, Norway \\ ${ }^{5}$ Swedish Institute of Space Physics, Uppsala, Sweden \\ ${ }^{6}$ EISCAT Scientific Association, Longyearbyen, Norway \\ ${ }^{7}$ Rutherford Appleton Laboratory, UK \\ ${ }^{8}$ Dept. of Physics, University of Troms $\varnothing$, Troms $\varnothing$, Norway \\ ${ }^{9}$ Remspace Inc., Linköping, Sweden \\ ${ }^{10}$ Oulo University, Oulu, Finland
}

Received: 14 July 2004 - Revised: 14 April 2005 - Accepted: 3 May 2005 - Published: 28 July 2005

\begin{abstract}
The shape of the electron energy distribution has long been a central question in the field of highfrequency radio-induced optical emission experiments. This report presents estimates of the electron energy distribution function, $f_{e}(E)$, from 0 to $60 \mathrm{eV}$, based on optical multiwavelength $(6300,5577,8446,4278 \AA$ ) data and $930-\mathrm{MHz}$ incoherent scatter radar measurements of ion temperature, electron temperature and electron concentration. According to our estimate, the electron energy distribution has a depression at around $2 \mathrm{eV}$, probably caused by electron excitation of vibrational states in $\mathrm{N}_{2}$, and a high energy tail that is clearly supra-thermal. The temporal evolution of the emissions indicates that the electron temperature still plays an important role in providing electrons with energies close to $2 \mathrm{eV}$. At the higher energies the electron energy distribution has a nonthermal tail.
\end{abstract}

Keywords. Active experiments; Ionosphere atmosphere interaction; Ionospheric physics

\section{Introduction}

Ionospheric high frequency $(\mathrm{HF}, 3-30 \mathrm{MHz})$ radio waveinduced optical emissions have been studied since the early 1970s (Biondi et al., 1970; Haslett and Megill, 1974) at low (Bernhardt et al., 1988), middle (Bernhardt et al., 1989a) and auroral (Brändström et al., 1999; Kosch et al., 2000; Pedersen and Carlson, 2001) latitudes. Even so, little is known about

Correspondence to: B. Gustavsson

(bjorn.gustavsson@nipr.ac.jp) the mechanism by which energy from the HF radio wave is transferred to the excitations of the atoms, molecules and ions that emit the light. One major step to clarify this issue is to determine the shape of the electron energy distribution function $f_{e}(E)$ during HF-pumping. In this report, we estimate the shape of the electron energy distribution using European Incoherent Scatter (EISCAT) UHF measurements of electron concentration, $n_{e}$, and temperature, $T_{e}$, and optical observations by the Auroral Large Imaging System (ALIS) (Steen et al., 1997; Brändström, 2003) and Digital All-Sky Imager (DASI) (Kosch et al., 1998) of enhanced emissions at 4278, 5577, 6300 and $8446 \AA$.

When a powerful HF radio wave is transmitted into the ionosphere with a frequency less than the peak ionospheric plasma frequency, this wave deposits most of its energy into the plasma in a narrow region. This region ranges from the reflection altitude, where the pump frequency equals the local plasma frequency, $f_{P}$, down to the altitude of upper hybrid resonance, where the pump frequency, $f_{0}$, equals the local upper hybrid frequency $\left.f_{U H}=\left(f_{\gamma}^{2}+f_{P}^{2}\right)^{1 / 2}\right)$, and at times several kilometers below. This process is intricate, primarily for the following three reasons: the energy of the pump wave is much less than the thermal energy of the electrons; the plasma is weakly ionized; and there are several possible nonlinear physical processes that can contribute. Moreover, these processes might contribute in different proportions depending on whether $f_{0}$ is above, below or within a few $\mathrm{kHz}$ of a multiple of the electron gyro resonance $\left(f_{0} \approx n f_{\gamma}\right)$ at the altitude where $f_{0}=f_{U H}$. 

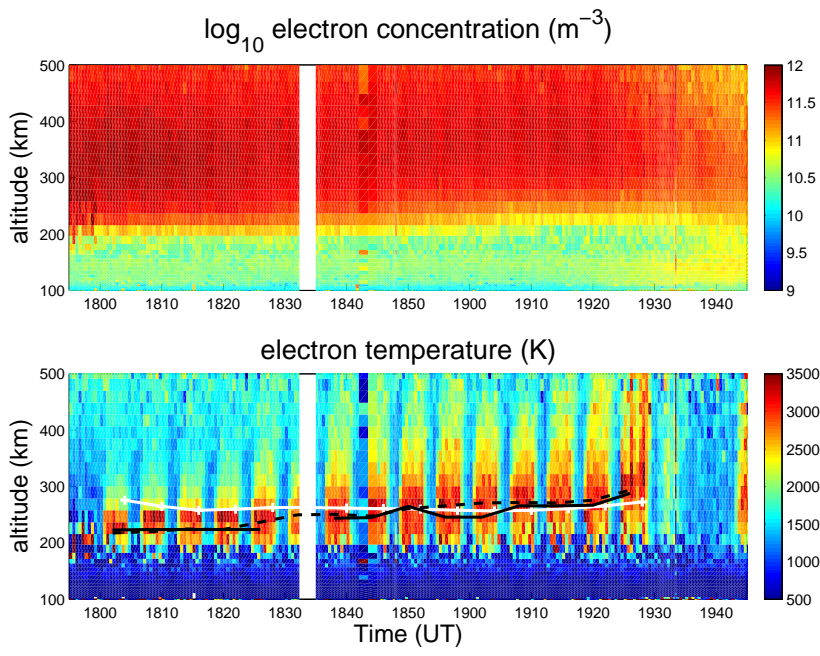

Fig. 1. EISCAT UHF observations of the electron concentration (top panel) and electron temperature (bottom panel). As seen in the top panel the electron concentration in the F region decays steadily, and there are no signs of significant precipitation until 19:30 UT. The electron temperature reaches $3000-3500 \mathrm{~K}$ for each pulse. For the first pulses the hot $\left(T_{e}>2500 \mathrm{~K}\right)$ region is approximately $50 \mathrm{~km}$ in altitude extent but gradually the heated region extends to more than $100 \mathrm{~km}$. The dashed black line is the approximate upper hybrid level, the solid black line is the altitude of the maximum temperature enhancements and the white line is the altitude where an MSIS model atmosphere gives the same $O\left({ }^{1} D\right)$ effective lifetime, as estimated from the DASI $6300 \AA$ observations.

Early observations of high ratios between enhancements in 5577 and $6300 \AA$ presented by Haslett and Megill (1974) and Bernhardt et al. (1989b) have been taken as an indication that the electron distribution causing the enhanced emissions has a significant accelerated part (e.g. Bernhardt et al., 1989b; Gurevich et al., 1985). Some experimental indications of accelerated electrons from Arecibo (IS-radar) have been presented (Carlson et al., 1982; Gurevich et al., 2000). This has been explained with electron acceleration by Langmuir turbulence (LT) excited at the reflection altitude. Specifically, weak LT has been dealt with theoretically by, for example, Perkins and Kaw (1971), Weinstock (1975), and Weinstock and Bezzerides (1974), and strong LT by DuBois et al. (1990) and Gurevich et al. (1985). Numerical simulations for ionospheric conditions have yielded power-law like tails of the electron distribution (Wang et al., 1997). Others (primarily Mantas, 1994; Mantas and Carlson, 1996) argued that the enhancements in $6300 \AA$ could well be explained by excitation of the high energy tail of the heated electron distribution and that the early observations of enhancements in $5577 \AA$ were scarce and unreliably close to the instrumental noise level.

With improved optical instrumentation, recent observations (e.g. Gustavsson et al., 2003) show that the enhancements at $5577 \AA$ regularly reach $10-30 \%$ of the $6300 \AA$ enhancements. This reinforces the view that a significant part of the enhanced emissions are caused by non-thermal electrons. Further, optical and incoherent scatter radar observa- tions coupled with thermosphere-ionosphere modeling have shown that the thermal model overestimates the $6300 \AA$ enhancements (Sergienko et al., 2000; Gustavsson et al., 2001). This decrease in excitation of the $O\left({ }^{1} D\right)$ state can be explained by a depletion in the electron energy distribution from 2 to some $6 \mathrm{eV}$ caused by the interaction of the electrons with the much more abundant neutrals (typical ionto-neutral ratios in these nighttime experiments range from $10^{-4}$ to $10^{-9}$ ) in the F region (Mishin et al., 2000; Gustavsson et al., 2004). However, this effect cannot explain the large 5577 to 6300 ratio.

Other observations show a minimum in the enhancements in $6300 \AA$ (Kosch et al., 2002; Bernhardt et al., 2000) when $f_{0}$ is at an electron gyro resonance at the altitude where $f_{0}=f_{U H}$. During these conditions, UH and electron Bernstein phenomena are suppressed, as are anomalous absorption and electron heating. This allows more of the pump wave energy to propagate to the pump reflection altitude. Despite the larger amount of energy available to excite LT, no significant enhancements at $6300 \AA$ are produced. This indicates that LT alone is not the dominant cause of enhanced optical emissions.

\section{Experiment and data}

From 18:00 to 19:36 UT on 10 March 2002, the EISCAT Heating Facility (Rietveld et al., 1993) in Troms $\varnothing\left(69.6^{\circ} \mathrm{N}\right.$, $19.2^{\circ} \mathrm{E}$ ) was operated at $5.423 \mathrm{MHz}$, transmitting $\sim 375 \mathrm{MW}$ effective radiated power in ordinary mode with a 4 min on and 2 min off cycle to enhance airglow in the nighttime $F$ region ionosphere. The antenna beam was approximately $6.8^{\circ}$ wide and directed $11.8^{\circ}$ south of zenith. During the entire experiment the EISCAT UHF incoherent scatter radar measured electron temperature $\left(T_{e}\right)$ enhancements up to 3000 $3500 \mathrm{~K}$, while the $\mathrm{F}$ region electron density decayed, as shown in Fig. 1. The temperatures seen agree well with previous observations (Gustavsson et al., 2001; Rietveld et al., 2003; Leyser et al., 2000), even though the effective radiated power here is approximately 2 times greater. Two factors that contribute to limiting the temperature are that the energy transfer from the electrons increases exponentially with temperature, and that the HF pump deposits its energy where the electron density is 1.7 times higher when transmitting at $5.423 \mathrm{MHz}$ compared to $4.04 \mathrm{MHz}$.

During this period the Auroral Large Imaging System (ALIS) imager temporarily located in Skibotn $\left(69.35^{\circ} \mathrm{N}\right.$, $20.36^{\circ} \mathrm{E}$ ) made observations in the $6300,5577,8446$ and $4278 \AA$ emissions with 7-s exposure every 9-11 s, switching filters between every image. As can be seen in Fig. 2 the full-width at half-maximum of the region with enhanced emissions is approximately $13 \pm 1 \mathrm{~km}$ and equal for the 5577 and $8446 \AA$ lines. Due to diffusion and drift the full-width at half-maximum for the $6300 \AA$ is slightly larger at $15 \pm 2 \mathrm{~km}$. For the $4278 \AA$ emission the size is smaller at $10 \pm 4 \mathrm{~km}$ but it might only appear so because the enhancement is close to the noise level. 

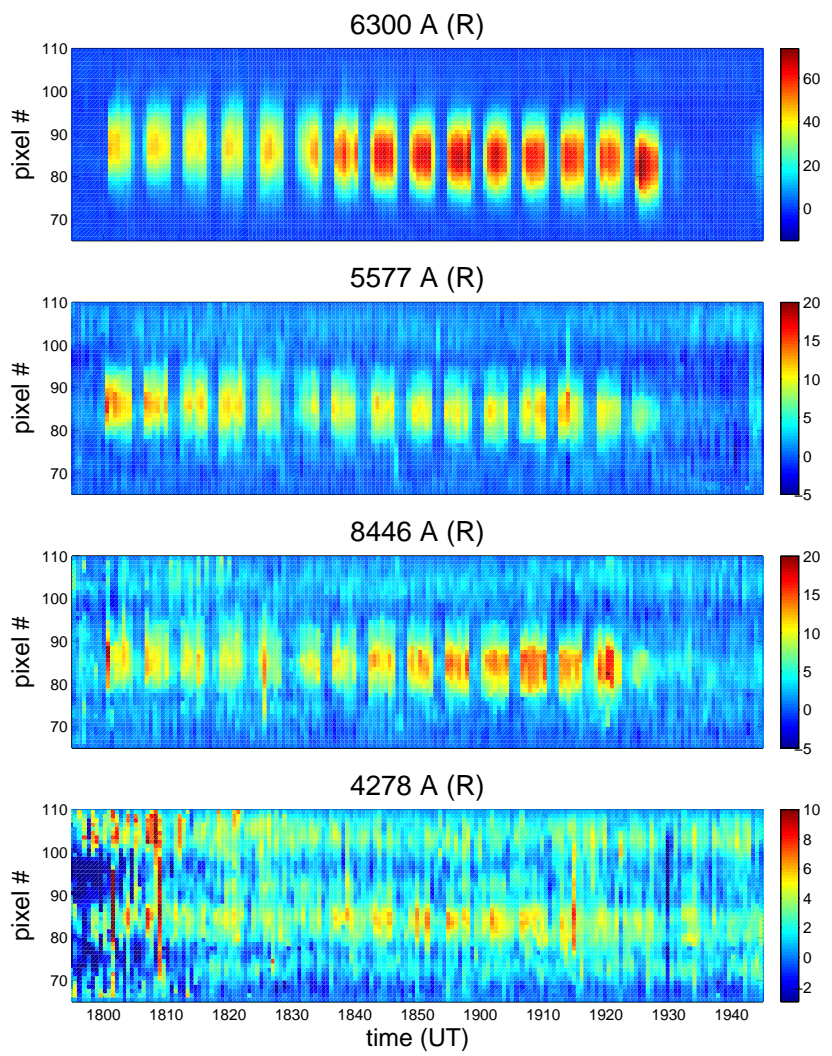

Fig. 2. Keograms of the ALIS observations after background subtraction, in the top panel the enhancements in $6300 \AA$, in the second panel $5577 \AA$, in the third $8446 \AA$ and in the fourth $4278 \AA$. The scale on the vertical axis is pixel number, the width of the red-line enhancement is approximately $3^{\circ}$ which corresponds to a $15-\mathrm{km}$ width at $250 \mathrm{~km}$ altitude. The enhancement in $6300 \AA$ is $40 \pm 3 \mathrm{R}$ for the first pulses then, with the rise in altitude and widening of the heated electrons, it increases to 55-70 R. For the $5577 \AA$ the enhancement for the first two pulses are $10-15 \mathrm{R}$ and then it varies around $10 \mathrm{R}$. For $8446 \AA$ the enhancement increases from $10 \pm 2$ to $15 \pm 3 \mathrm{R}$ and for the $\left.4278 \AA N_{2}^{+}(1 N G)\right)$ emission the enhancements are indistinguishable from the noise for the first pulses but they grow up to $5 \mathrm{R}$ at $18: 42 \mathrm{UT}$.

Simultaneously, the co-located digital all-sky imager (DASI) made observations in the $6300 \AA$ emission from $O\left({ }^{1} D\right)$ from $18: 00$ to $18: 30 \mathrm{UT}$, from $18: 50$ to $19: 06 \mathrm{UT}$ and from 19:26 to $19: 34 \mathrm{UT}$; and in the $5577 \AA$ emission from $O\left({ }^{1} S\right)$ from 18:33 to $18: 47 \mathrm{UT}$ and from 19:09 to 19:23 UT, all with $10 \mathrm{~s}$ exposure and 1 image every 10-s. As can be seen in Fig. 3 the intensity grows from around $10 \mathrm{R}$ for the first pulse to $60-70 \mathrm{R}$ for the pulses after 18:48 UT. From the initial decay of the $6300 \AA$ emission, we estimate the $O\left({ }^{1} D\right)$ effective lifetime $\tau_{O\left({ }^{1} D\right)}$ to be 26-33 s, using the method suggested by Bernhardt et al. (2000). The results are listed in Table 1.

Other measurements which were made during the experiment, but are not used in this study, included power and spectra of Langmuir turbulence using the EISCAT $224 \mathrm{MHz}$ incoherent scatter radar, and stimulated electromagnetic emis-
Table 1. Estimates of $O\left({ }^{1} D\right)$ effective lifetime from the initial decay of the enhancement in $6300 \AA$ emission as observed by DASI.

\begin{tabular}{cc}
\hline HF-pulse $(\mathrm{UT})$ & $\tau_{O}\left({ }^{1} D\right)(\mathrm{s})$ \\
\hline $18: 10$ & 29 \\
$18: 16$ & 26 \\
$18: 22$ & 26 \\
$18: 28$ & 28 \\
$18: 52$ & 27 \\
$18: 58$ & 26 \\
$19: 06$ & 25 \\
$19: 28$ & 33 \\
\hline
\end{tabular}

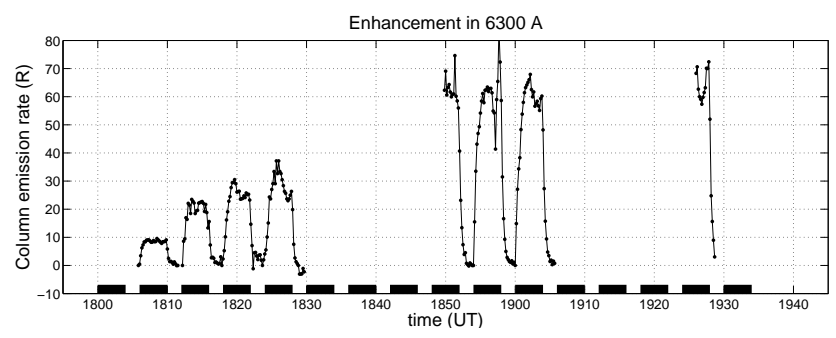

Fig. 3. Maximum enhancements in $6300 \AA$ after background reduction. The black squares mark the HF-pump-periods.

sions around the HF pump frequency, using a spectrum analyzer $15 \mathrm{~km}$ east of the Heating facility.

\section{Data analysis}

To estimate $f_{e}(E)$ we look at the contribution to the emissions from energetic electrons. For $I_{5577}$ there are two main sources of $O\left({ }^{1} S\right)$. The most important in the F region is direct electron excitation:

$O+e^{-} \rightarrow O\left({ }^{1} S\right)+e^{-}$,

which has an excitation threshold of $4.17 \mathrm{eV}$ and a cross section as depicted in Fig. 4. The second is energy transfer from $N_{2}\left(A^{3} \Sigma_{u}^{+}\right)$

$N_{2}\left(A^{3} \Sigma_{u}^{+}\right)+O \rightarrow N_{2}+O\left({ }^{1} S\right)$,

which does not give a significant contribution in the $\mathrm{F}$ region where the concentration of $N_{2}$ is much lower than $\mathrm{O}$.

For $I_{6300}$ the major sources from energetic electrons to $O\left({ }^{1} D\right)$ are direct electron excitation

$O+e^{-} \rightarrow O\left({ }^{1} D\right)+e^{-}$,

with an excitation threshold of $1.96 \mathrm{eV}$ and about a 10 times higher cross section than that for $O\left({ }^{1} S\right)$ and cascading from $O\left({ }^{1} S\right)$

$O\left({ }^{1} S\right) \rightarrow O\left({ }^{1} D\right)+h v_{\lambda=5577 \AA}$. 


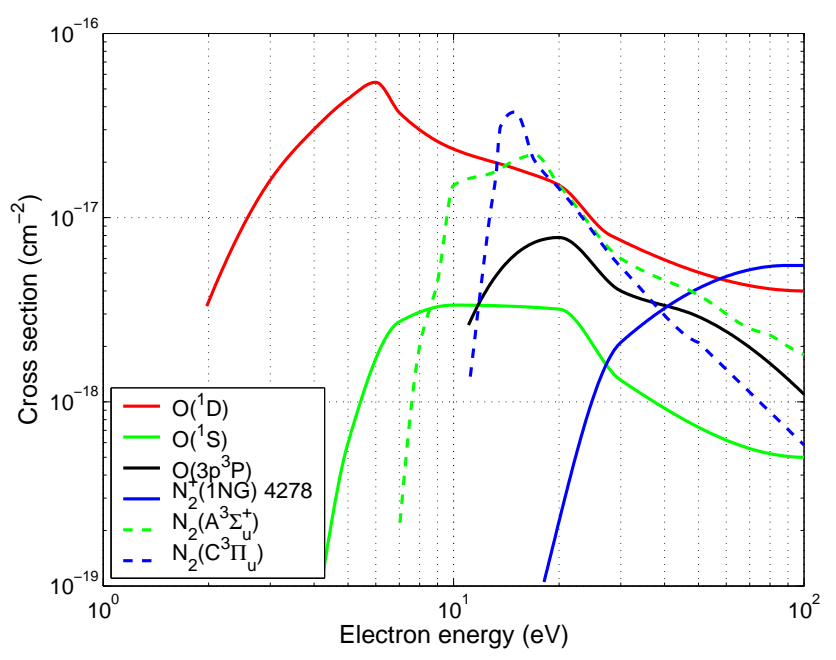

Fig. 4. Cross sections for electron impact excitation of $O\left({ }^{1} D\right)-$ emitting at $6300 \AA$, with excitation threshold $1.965 \mathrm{eV}$ - experimental data from Doering (1992), $O\left({ }^{1} S\right)$ - emitting at $5577 \AA$, with excitation threshold $4.19 \mathrm{eV}$ - experimental data from Doering and Gulcicek (1989), $O\left(3 p^{3} P\right)-$ emitting at $8446 \AA$, with excitation threshold $10.99 \mathrm{eV}$ - experimental data from Itikawa and Ichimura (1990). For electron excitation of $\left(N_{2}\right)$ we have the emission cross section for $4278 \AA$ from $\left(N_{2}^{+}\right)$with threshold for ionization + excitation at $18 \mathrm{eV}$ and excitation cross section for $N_{2}^{+}\left(A^{3} \Sigma_{u}^{+}\right)$and $N_{2}\left(C^{3} \Pi_{u}\right)-$ all with experimental data from Itikawa et al. (1986).

In the quiet F-region ionosphere the major source of $O\left({ }^{1} D\right)$ is excitation from dissociative recombination of $\mathrm{O}_{2}^{+}$.

$\mathrm{O}_{2}^{+}+e^{-} \rightarrow O\left({ }^{1} D\right)+O$

with a yield, $\mathrm{Q}$, of 1.2. For situations with rapid variations this has to be included since its rate varies with $T_{e}$

$k=1.9^{-7}\left(300 / T_{e}\right)^{0.5}$.

However, at steady state, which we considers here, the loss of $\mathrm{O}_{2}^{+}$is balanced by the source-charge transfer from $\mathrm{O}^{+}$to $\mathrm{O}_{2}$

$\mathrm{O}^{+}+\mathrm{O}_{2} \rightarrow \mathrm{O}+\mathrm{O}_{2}^{+}$

Thus, we obtain an $O\left({ }^{1} D\right)$ excitation rate that is independent of $T_{e}$

$r_{O}\left({ }^{1} D\right)=n_{O_{2}^{+}} n_{e} k Q=n_{O_{2}^{+}} n_{e} k=n_{O^{+}} n_{O_{2}} K$
$\left(n_{O^{+}} n_{O_{2}} K / n_{e} k\right) n_{e} k Q=n_{O^{+}} n_{O_{2}} K Q$.

According to Chen et al. (1978) the rate coefficient $(K)$ for charge transfer from $\mathrm{O}^{+}$is to $\mathrm{O}_{2}$

$K=3.33 \times 10^{-12} \exp \left(3.72\left(300 . / T_{f}\right)-1.78\left(300 . / T_{f}\right)^{2}\right)$

where $T_{f}$ is the average of the neutral and ion temperatures. At the end of the $4 \mathrm{~min}$ HF pump periods the modified $O\left({ }^{1} D\right)$ excitation rates from dissociative recombination is within a few percent of the natural background rates.
There are two sources for I $_{8446}$ : electron excitation of atomic oxygen and dissociative excitation of molecular oxygen. Since the concentration of molecular oxygen is approximately $5 \%$ of the atomic oxygen at the region of interest and the emission cross section for dissociative excitation is a factor of 5 smaller, we only include direct excitation of atomic oxygen. Finally, the only source of $I_{4278}$ is electron impact ionization to the $N_{2}^{+}\left(B^{2} \Sigma_{u}^{-}\right)$state, for which we use the emission cross section from Itikawa et al. (1986). However, about half the intensity of the $1-5 N_{2} 2 P$ band at $4268 \AA$ passes through the passband of the filter. Thus, we have to take that into account. For the $N_{2}\left(C^{3} \Pi_{u}\right)$ we obtain the excitation cross section from Itikawa et al. (1986) and use Einstein coefficients and Franck-Condon factors from Gilmore et al. (1992).

In order to obtain a simple set of equations for $f_{e}(E)$ we note that the region of enhanced emission is small, with a typical size of about $30 \mathrm{~km}$ (Gustavsson et al., 2001), and assume that all excitation occurs in a $10-\mathrm{km}$ wide region with conditions for the HF-pump wave reflection altitude. This gives us the following set of integral equations for the column emission rates:

$$
I_{5577}=10^{-6} \frac{A_{5577}}{\sum A_{O\left({ }^{1} S\right)}} n_{O} \Delta z \int_{0}^{\infty} v_{e}(E) \sigma_{2}(E) f_{e}(E) d E
$$

$$
\begin{gathered}
I_{6300}=10^{-6} A_{6300} \tau_{O}^{I D}\left(I_{5577}+n_{O} \Delta z\right] \\
\left.\int_{0}^{\infty} v_{e}(E) \sigma_{1}(E) f_{e}(E) d E\right),
\end{gathered}
$$

$I_{8446}=10^{-6} n_{O} \Delta z \int_{0}^{\infty} v_{e}(E) \sigma_{3}(E) f_{e}(E) d E$,

$I_{4278}=10^{-6} n_{N_{2}} \Delta z \int_{0}^{\infty} v_{e}(E) \sigma_{4}(E) f_{e}(E) d E$,

$I_{4268}=10^{-6} n_{N_{2}} Q \Delta z \int_{0}^{\infty} v_{e}(E) \sigma_{5}(E) f_{e}(E) d E$.

Here $A_{i}$ is the Einstein coefficient for the emission, listed in Table 2, and the sum $\sum A_{i}^{\prime}(z)$ is taken over all loss processes for the emitting state. (Q) in Eq. (14) is $\left(q_{X^{-} C} A_{i, 1-5} \sum A_{i, 1-j}=0.308 \cdot 8.98 \cdot 10^{5} / 2.66 \cdot 10^{7}\right)$, with values of Franck-Condon factors and Einstein coefficients from Gilmore et al. (1992).

Furthermore, $n_{j}$ is the local concentration of the emitting species, $\sigma_{i}$ is the excitation cross section, $v_{e}$ is the electron velocity, $f_{e}(E, z)$ is the electron energy distribution as a function of energy $(E)$ and altitude $(z)$. For the neutral densities we use the MSIS model (Hedin, 1991) at the altitude of interaction. 
Table 2. Transition probabilities from $O\left({ }^{1} S\right)$ and $O\left({ }^{1} D\right)$. Einstein coefficients recommended by Itikawa and Ichimura (1990).

\begin{tabular}{ll}
\hline Transition & Einstein coeff $\left(s^{-1}\right)$ \\
\hline $\mathrm{O}\left({ }^{1} D\right)-\mathrm{O}\left({ }^{3} P\right)_{2}$ & $5.627 \cdot 10^{-3}$ \\
$\mathrm{O}\left({ }^{1} D\right)-\mathrm{O}\left({ }^{3} P\right)_{1}$ & $1.818 \cdot 10^{-3}$ \\
$\mathrm{O}\left({ }^{1} D\right)-\mathrm{O}\left({ }^{3} P\right)_{3}$ & $8.922 \cdot 10^{-7}$ \\
& \\
$\mathrm{O}\left({ }^{1} S\right)-\mathrm{O}\left({ }^{3} P\right)_{2}$ & $2.732 \cdot 10^{-4}$ \\
$\mathrm{O}\left({ }^{1} S\right)-\mathrm{O}\left({ }^{3} P\right)_{1}$ & $7.601 \cdot 10^{-2}$ \\
$\mathrm{O}\left({ }^{1} S\right)-\mathrm{O}\left({ }^{1} D\right)$ & 1.215 \\
\hline
\end{tabular}

Equations (10) to (14) are a set of integral equations for $f_{e}(E)$ that we solve by searching for optimal parameters of the following parameterization of $f_{e}(E)$ :

$$
\frac{f_{e}(E)}{E^{1 / 2}} \propto\left\{\begin{array}{cc}
n_{0} e^{\frac{-E}{k_{B} T_{e}}} & E<=E_{1} \\
\max \left(n_{2}\left(\frac{E}{E_{0}}\right)^{\gamma}, n_{1} e^{\frac{-E}{k_{B} T_{1}}}+\right. & \\
\left.n_{2} e^{-\frac{\left(E-E_{0}\right)^{2}}{d E^{2}}}\right) & E_{1}<E<E_{0} \\
n_{1} e^{\frac{-E}{k_{B} T_{1}}}+n_{2} e^{-\frac{\left(E-E_{0}\right)^{2}}{d E^{2}}} & E>E_{0} .
\end{array}\right.
$$

Here $E_{1}=1.96 \mathrm{eV}$ and we take $\left(n_{1}, T_{1}, n_{2}, E_{0}, d E, \gamma\right)$ as free parameters and determine $n_{0}$ to give an electron concentration according to the EISCAT UHF observations.

This parameterization allows us to obtain a wide variety of electron energy distributions. By separating the number of electrons in the tail (by using $n_{1}$ and $n_{2}$ for the electrons with $E>2 \mathrm{eV}$ and $n_{0}$ for the bulk electrons) we can roughly model the electron "bite-out" at $2 \mathrm{eV}$. This modification is caused mainly by energy loss to vibrational states of $\mathrm{N}_{2}$, and $f_{e}(E)$ is estimated to be depleted to $10-20 \%$ in the lower $F$ region (Mishin et al., 2000). The reason that we require that $\gamma<1 / 2$ is to avoid "bump-on-tail" populations. That HF-pumping cannot produce "bump-on-tail" populations is a reasonable assumption, considering that the energy of the pump wave is much less than the thermal energy of the electrons. In order to obtain a good fit with the observed emissions it was not enough to assume a power-law tail, and thus we had to add the accelerated population $\left(n_{2} e^{-\left(E-E_{0}\right)^{2} / d E^{2}}\right)$ and the "higher temperature in the tail than the bulk" electrons $\left(n_{1} E^{1 / 2} e^{-E / k_{B} T_{1}}\right)$. This parameterization should capture the major features of the modified electron energy distribution. However, keeping in mind the complexity of electron energy distributions in the auroral ionosphere, we are aware that the real modified electron energy distribution might be more complex.

The electron energy distribution is obtained by minimization of the difference between the observed intensities and the intensities predicted by Eqs. (10) to (14). As can be seen in Fig. 5 it is possible to obtain a good agreement between the observed data and modeled emission enhancements.

The reason that we obtain such a perfect fit for almost all pulses is that there are six degrees of freedom in the parame-

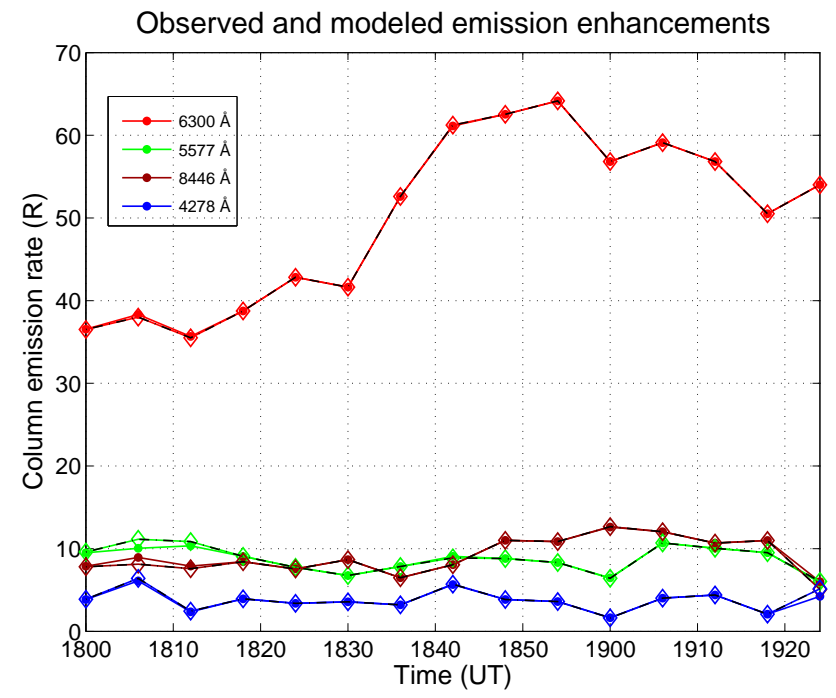

Fig. 5. Modeled electron enhanced emission rates, marked with diamonds and dot-dashed lines, agree well with the observed enhancements, marked with dots. The fit between observed and modeled emissions is almost perfect. For the 6300 and 8446 lines the differences are noticeable only for the second pulse. For 5577 it is possible to see deviations for the second and third pulse. For 4278 , only the last pulse has an error large enough to be distinguishable.

terization and only four equations. However, it is worth noting the constraints that we impose - that the flux of energized electrons at $100 \mathrm{eV}$ is negligible and that the discontinuity a $2 \mathrm{eV}$ corresponds to a depletion - makes the problem solvable. Further, the parameterization is in its structure very smooth which helps to avoid problems common in under-determined problems - noise sensitivity, and rapid fluctuations. To estimate the stability of the solution we also start the optimization at a large number of points in the parameter space, and it appears that the numerical optimization gives approximately the same results for most of the starting points.

\subsection{Interpretation of fitted parameters}

The typical shape of the estimated electron energy distribution from 2 to $100 \mathrm{eV}$ has a thermal-like part from $2 \mathrm{eV}$ up to some $10-20 \mathrm{eV}$, then a small but significant accelerated tail with an acceleration energy of between 40 and $60 \mathrm{eV}$ and a sharp cutoff at higher energies, as shown in Fig. 6. Here it should be noted that even though the electron distribution has a positive slope tail, the electron distribution function is flat.

At energies from $2 \mathrm{eV}$ to $12-30 \mathrm{eV}$ all but one electron energy distribution (19:00-19:04 UT) have thermal-like components with temperatures ranging from 12000 to $47000 \mathrm{~K}$.

As can be seen in Fig. 7 this temperature is much larger than the bulk temperature measured by EISCAT UHF. However, the fraction of electrons in this super-hot tail is very small, which is accentuated by the depletion in $f_{e}(E)$ at $2 \mathrm{eV}$, caused mainly by excitation of the vibrational states in $N_{2}$. 


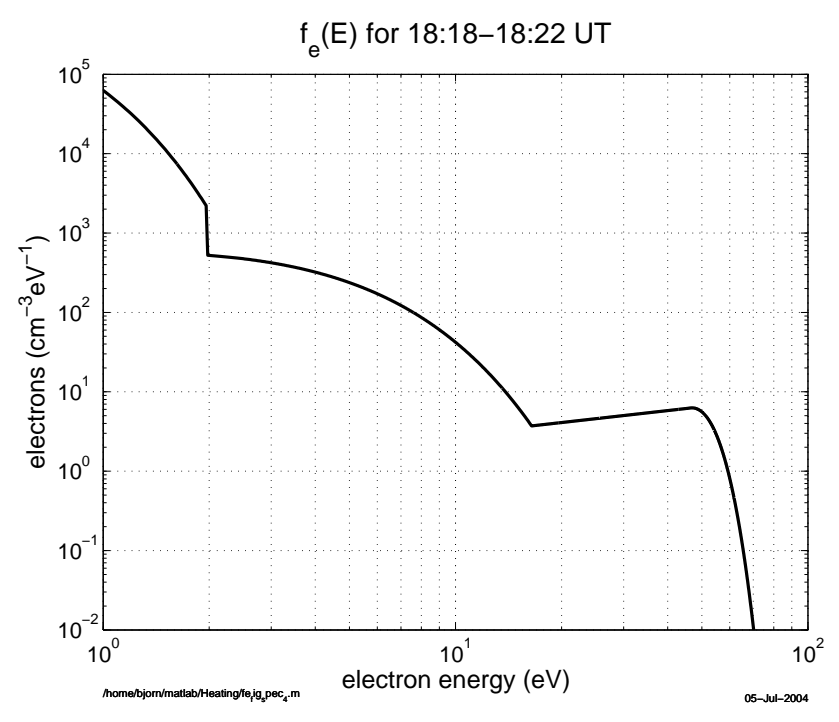

Fig. 6. Electron energy distribution as a function of energy.
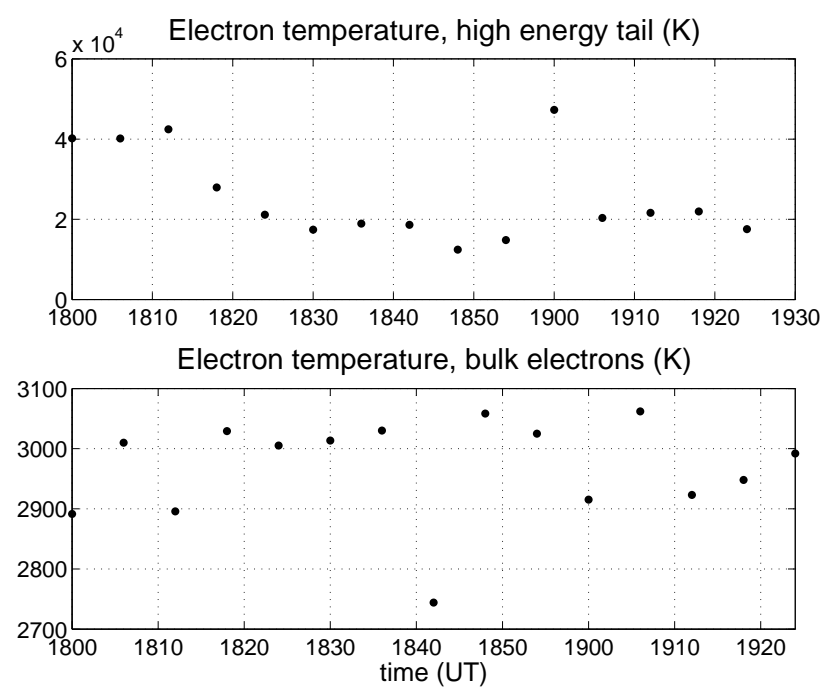

Fig. 7. Upper panel shows the electron temperature in the high energy tail, from $2 \mathrm{eV}$ to approximately $20 \mathrm{eV}$. For comparison the lower panel displays the EISCAT UHF measurements of $\left(T_{e}\right)$ at steady state in the region of highest temperature.

For the period from 18:00 to 18:40 UT this depletion decreases from over $80 \%$ during the first pulses to less than $1 \%$ around 19:00 UT, as is shown in Fig. 8. For the later period the depletion is again larger by 30 to $50 \%$.

The parameter $\gamma$ is approximately $1 / 2$ for all but four HF pump pulses. This corresponds to an electron distribution function that is flat $\left(f(\bar{v})=C, E \min <m_{e} \bar{v}^{2} / 2<E 0\right)$ in the tail from $12-22 \mathrm{eV}$ to the acceleration energy, as can be seen in Fig. 9. For the three pulses (18:48-18:52, 18:5418:56 and 19:18-19:22 UT) with a power-law component from $9-11 \mathrm{eV}$ to the acceleration energy the exponent $\gamma$ in $\left(f_{e}(E) \propto\left(E / E_{0}\right)^{\gamma}\right)$ is $-0.5,-0.04$ and -1.23 respectively. The results here do not, by and large, indicate electron distri-

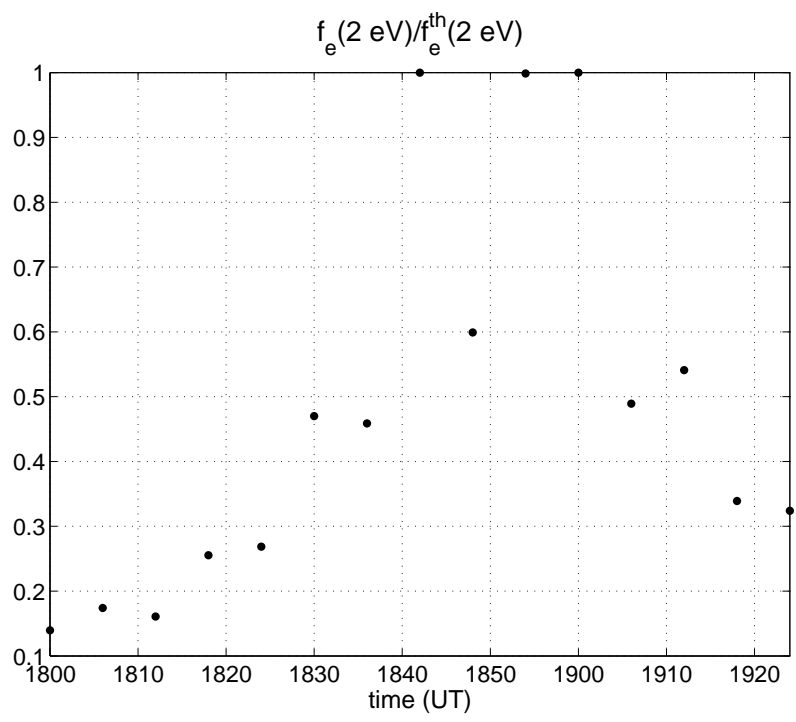

Fig. 8. Estimate of the ratio of $f_{e}(E) / f_{e}^{t h}(E)$ at $2 \mathrm{eV}$. Here $f_{e}^{t h}(E)$ denotes a thermal energy distribution. Note that the depletion is significant for the first five pulses when the approximate altitude of upper hybrid resonance is below $250 \mathrm{~km}$ and varies for the last pulses when the interaction altitude is above $250 \mathrm{~km}$. This is consistent with the decrease in neutral concentrations with increasing altitudes.

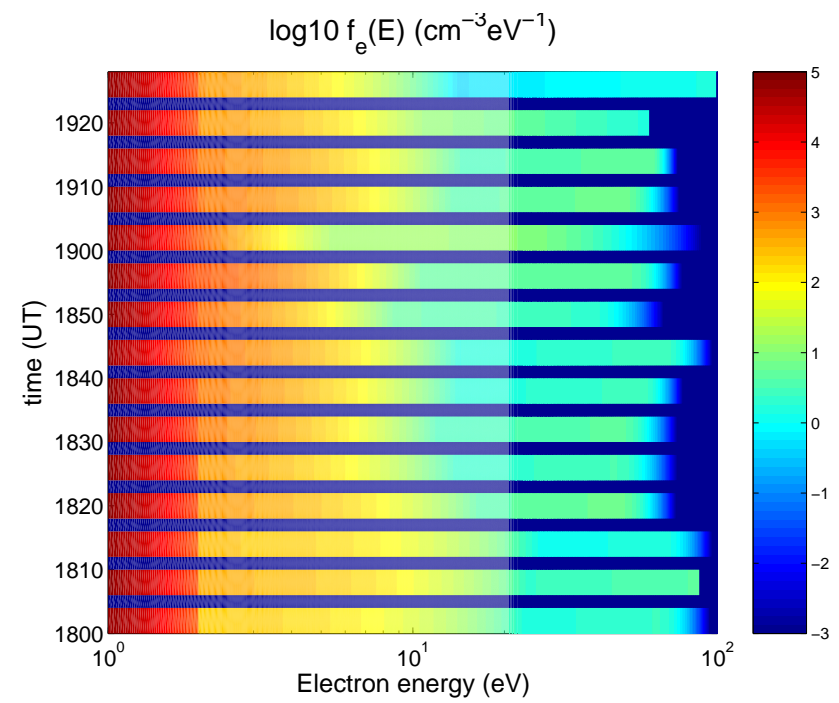

Fig. 9. Electron energy distribution as a function of energy and time.

butions dominated by power-law tails, as has been suggested by Mishin et al. (e.g. 2003).

\section{Summary}

In this report we have presented incoherent scatter and optical data from a HF pump experiment where enhanced optical emission in 6300,5577 and $8446 \AA$ from atomic oxygen and $4278 \AA$ from $N_{2}^{+}$was observed, as well as electron 
temperature enhancements of up to $3500 \mathrm{~K}$. Further, we have developed a method to estimate the shape of the electron energy distribution function in the energy range from 1.96 to $100 \mathrm{eV}$. The resulting estimates of the electron energy distribution show that there is an accelerated component with energies from $15-22 \mathrm{eV}$ to $45-65 \mathrm{eV}$ where the electron distribution is essentially flat. At intermediate energies below $15 \mathrm{eV}$ and above 1.96 the electron energy distribution is most often thermal-like with temperatures between 9000 and $43000 \mathrm{~K}$. At $2 \mathrm{eV}$ there seems to be a depletion of between 10 and $90 \%$ in the electron energy distribution. Gurevich and Milikh (1997) and Bernhardt et al. (1989b) have suggested that the super-heated component in the electron energy distribution explains the high ratio between enhancements in 5577 and $6300 \AA$, which is in agreement with the observations presented in Gustavsson et al. (2003). Mishin et al. (2000) have predicted the depletion at $2 \mathrm{eV}$ to be of the magnitude observed here during F-region HF-pump experiments. But in order to explain the observed enhancements in 8446 and $4278 \AA$ that we present here, there has to be a component of electron distribution accelerated to higher energies than that previously expected.

Acknowledgements. The authors thanks the unknown referees for their valuable comments.

Topical Editor M. Lester thanks E. Mishin for his help in evaluating this paper.

\section{References}

Steen, ̊., Brändström, U., and Gustavsson, B.: ALIS — a multi station imaging system at high latitude with multi disciplinary scientific objectives, in Proceedings 13th ESA Symposium on European Rocket and Balloon Programmes and Related Research, pp. 261-266, ESA, ESA SP-397, 1997.

Bernhardt, P. A., Duncan, L. M., and Tepley, C. A.: Artificial airglow excited by high-power radio wawes, Science, 242, 10221027, 1988.

Bernhardt, P. A., Duncan, L. M., and Tepley, C. A.: Heater induced cavities as optical tracers of plasma drift, J. Geophys. Res., 94, 7003-7010, 1989a.

Bernhardt, P. A., Tepley, C. A., and Duncan, L. M.: Airglow enhancements associated with plasma cavities formed during ionospheric heating experiments, J. Geophys. Res., 94, 9071-9092, 1989b.

Bernhardt, P. A., Wong, M., Huba, J. D., Fejer, B. G., Wagner, L. S., Goldstein, J. A., Selcher, C. A., Frolov, V. L., and Sergeev, E. N.: Optical remote sensing of the thermosphere with HF pumped artificial airglow, J. Geophys. Res., 105, $10657-10671,2000$.

Biondi, A. A., Sipler, D. P., and Hake, R. D.: Optical $(\lambda-6300)$ detection of radio frequency heating of electrons in the $\mathrm{F}$ region, J. Geophys. Res., 75, 6421-6424, 1970.

Brändström, B. U. E.: The Auroral Large Imaging System - Design, operation and scientific results, Ph.D. thesis, Swedish Institute of Space Physics, Kiruna, Sweden, Sci. Report 279, 2003.

Brändström, B. U. E., Leyser, T. B., Steen, Å., Rietveld, M. T., Gustavsson, B., Aso, T., and Ejiri, M.: Unambigous evidence of HF pump-enhanced airglow, Geophys. Res. Lett., 26, 35613564, 1999.
Carlson, H. C., Wickwar, V. B., and Mantas, G. P.: Observations of fluxes of suprathermal electrons accelerated by HF excited instabilities, J. Atmos. Terr. Phys., 44, 1089-1100, 1982.

Chen, A., Johnsen, R., and Biondi, M. A.: Measurements of the $\left(\mathrm{O}^{+}+\mathrm{N}_{2}\right)$ and $\left(\mathrm{O}^{+}+\mathrm{O}_{2}\right)$ reaction rates from 300 to $900 \mathrm{~K}, \mathrm{~J}$. Chem. Phys., 69, 2688, 1978.

Doering, J. P.: Absolute Differential and Integral Electron Excitation Cross Section for Atomic Oxygen 9. Improved Cross Section for the ${ }^{3} P \rightarrow{ }^{1} D$ Transition From 4.0 to $30 \mathrm{eV}$, J. Geophys. Res., 97, 19531-19534, 1992.

Doering, J. P. and Gulcicek, E. E.: Absolute Differential and Integral Electron Excitation Cross Section for Atomic Oxygen 7. The ${ }^{3} P \rightarrow{ }^{1} D$ and ${ }^{3} P \rightarrow{ }^{1} S$ Transitions From 4.0 to $30 \mathrm{eV}, \mathrm{J}$. Geophys. Res., 94, 1541-1546, 1989.

DuBois, D., Rose, H. A., and Russel, D.: Caviton Dynamics in Strong Langmuir Turbulence, Physica Scripta, T30, 137-158, 1990.

Gilmore, F. R., Laher, R. R., and Espy, P. J.: Franck-Condon Factors, r-Centroids, Electronic Transition Moments, and Einstein Coefficients for Many Nitrogen and OPxygen Band Systems, J. Phys. Chem. Ref. Data, 21, 1005-1107, 1992.

Gurevich, A. V. and Milikh, G. M.: Artificial airglow due to modifications of the ionosphere by powerful radio waves, J. Geophys. Res., 102, 389-394, 1997.

Gurevich, A. V., Dimant, Y. S., Milikh, G. M., and Va'skov, V. V.: Multiple acceleration of electrons in the regions high power radio-wave reflection in the ionosphere, J. Atmos. Terr. Phys., 47, 1057-1070, 1985.

Gurevich, A. V., Carlson, H. C., Milikh, G. M., Zybin, K. P., Djuth, F. T., and Groves, K.: Suprathermal electrons generated by the interaction of powerful radio wave with the ionosphere, Geophys. Res. Lett., 27, 2461-2464, 2000.

Gustavsson, B., Sergienko, T., Rietveld, M. T., Honary, F., Steen, Å., Brändström, B. U. E., Leyser, T. B., Aruliah, A. L., Aso, T., and Ejiri, M.: First Tomographic estimate of volume distribution of enhanced airglow emission caused by HF pumping, J. Geophys. Res., 106, 29 105-29 123, 2001.

Gustavsson, B., Brändström, B. U. E., Steen, Å., Sergienko, T., Leyser, T. B., Rietveld, M. T., Aso, T., and Ejiri, M.: Nearly simultaneous images of HF-pump enhanced airglow at $6300 \AA$ and $5577 \AA$ Å, Geophys. Res. Lett., 29, doi:10.1029/2002GL015350, 2003.

Gustavsson, B., Sergienko, T., Häggström, I., and Honary, F.: Simulation of high energy tail of electron distribution function, Adv. Polar Upper Atmos. Res., 18, 1-9, 2004.

Haslett, J. C. and Megill, L. R.: A model of the enhanced airglow excited by RF-radiation, Radio Sci., 9, 1005-1019, 1974.

Hedin, A.: Extension of the MSIS Thermospheric Model into the Middle and Lower Atmosphere, J. Geophys. Res., 96, 1159$1172,1991$.

Itikawa, Y. and Ichimura, A.: Cross sections for collisions of electrons and photons with atomic oxygen, J. Phys. Chem. Ref. Data, 19, 637-651, 1990.

Itikawa, Y., Hayashi, M., Ichimura, A., Onda, K., Sakimoto, K., Takayanagi, K., Nakamura, M., Nishimura, H., and Takayanagi, T.: Cross sections for collisions of electrons and photons with nitrogen molecules, J. Phys. Chem. Ref. Data, 15, 985-1010, 1986.

Kosch, M., Hagfors, T., and Nielsen, E.: A new digital all-sky imager experiment for optical auroral studies in conjunction with the Scandinavian twin auroral radar experiment, Rev. Sci. Instrum., 69, 578-584, 1998. 
Kosch, M. J., Rietveld, M., T., Hagfors, T., and Leyser, T. B.: Highlatitude HF-induced airglow displaced equatorward of the pump beam, Geophys. Res. Lett., 27, 2817-2820, 2000.

Kosch, M. J., Rietveld, M. T., Kavanagh, A. J., Davis, C., Yeoman, T., Honary, F., and Hagfors, T.: High-latitude pump-induced optical emissions for frequencies close to the third electron gyroharmonic, Geophys. Res. Lett., 29, 27-1-274, 2002.

Leyser, T. B., Gustavsson, B., Brändström, B. U. E., Steen, Å., Honary, F., Rietveld, M. T., Aso, T., and Ejiri, M.: Simultaneous measurements of high-frequency pump-enhanced airglow and ionospheric temperatures at auroral latitudes, Adv. Polar Upper Atmos. Res., 14, 1-11, 2000.

Mantas, G. P.: Large 6300- $\AA$ airglow intensity enhancements observed in ionosphere heating experiments are exited by thermal electrons, J. Geophys. Res., 99, 8993-9002, 1994.

Mantas, G. P. and Carlson, H. C.: Reinterpretation of the 6300$\AA$ airglow enhancements observed in ionosphere heating experiments based on analysis of Platteville, Colorado, data, J. Geophys. Res., 101, 195-209, 1996.

Mishin, E., Carlson, H. C., and Hagfors, T.: On the Electron Distribution Function in the F Region and Airglow Enhancements During HF Modification Experiments, Geophys. Res. Lett., 27, 2857-2860, 2000.

Mishin, E., Burke, W. J., and Pedersen, T. R.: On the onset of HF-induced airglow at HAARP, J. Geophys. Res., 109, doi:10.1029/2003JA010205, 2003.

Pedersen, T. R. and Carlson, H. C.: First observations of HF heaterproduced airglow at the High frequency Active Auroral Research Program facility: Thermal excitation and spatial structuring, Radio Sci., 36, 1013-1026, 2001.
Perkins, F. W. and Kaw, P. K.: On the role of plasma instabilities in ionospheric heating by radio waves, J. Geophys. Res., 76, 282284, 1971.

Rietveld, M., Kosch, M., Blagoveshchenskaya, N., Kornienko, V., Leyser, T., and Yeoman, T.: Ionospheric electron heating, optical emissions and striations induced by powerful HF radio waves at high latitudes: Aspect angle dependence, J. Geophys. Res., 108, 1141-, 2003.

Rietveld, M. T., Kohl, H., Kopka, H., and Stubbe, P.: Introduction to ionospheric heating at Troms $\varnothing-\mathrm{I}$. Experimental overview, J. Atmos. Terr. Phys., 55, 577-599, 1993.

Sergienko, T., Gustavsson, B., Steen, Å., Brändström, U., Rietveld, M., Leyser, T., and Honary, F.: Analysis of excitation of the $630.0 \mathrm{~nm}$ airglow during heating experiment in Troms $\varnothing$ on 16 February 1999, Phys. Chem. Earth, 25, 531-535, 2000.

Wang, J. G., Newman, D. L., and Goldman, M. V.: Vlasov simulation of electron heating by Langmuir turbulence near the critical altitude in the radiation-modified ionosphere, J. Atmos. Solar and Terr. Phys., 59, 2461-2474, 1997.

Weinstock, J.: Theory of enhanced airglow during ionospheric modifications, J. Geophys. Res., 80, 4331-, 1975.

Weinstock, J. and Bezzerides, B.: Theory of electron acceleration during parametric instabilities, Phys. Rev. Lett., 32, 754-758, 1974. 